\title{
Atendimento multidisciplinar do paciente ortodôntico com epidermólise bolhosa
}

Patrícia Valéria Milanezi Alves*, Daniele Karina Milanezi Alves**, José Vinicius Bolognese Maciel*,

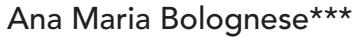

\begin{abstract}
Resumo
O termo epidermólise bolhosa descreve um grupo variado de doenças hereditárias, crônicas, não inflamatórias, epiteliais e da mucosa, que são caracterizadas por excepcional fragilidade e reduzida resistência após trauma moderado. Esta doença é classificada em forma simples, juncional ou distrófica, com pelo menos 23 subtipos, e é uma doença rara que afeta toda a população, qualquer grupo racial e igualmente homens e mulheres. Objetivo: os autores relatam problemas sistêmicos e bucais associados à epidermólise bolhosa, norteando atitudes clínicas multidisciplinares quando o paciente necessita de tratamento ortodôntico.
\end{abstract}

Palavras-chave: Epidermólise bolhosa. Ortodontia. Tratamento multidisciplinar.

\section{INTRODUÇÃO}

A epidermólise bolhosa congênita (EB) representa um grupo heterogêneo de patologias de caráter genético, caracterizadas pela formação de bolhas na região cutâneo-mucosa de todo o corpo. Fisiopatologicamente é causada pela adesão inadequada entre os componentes do epitélio e as mucosas, o que pode ocorrer em diferentes níveis, dependendo do subtipo em questão. Devido à pequena coesão e fragilidade mecânica, há formação de bolhas após trauma mecânico mínimo ${ }^{1,6,8}$.

Mais de 20 subtipos são descritos e classificados como forma simples, juncional ou distrófica, de acordo com o tipo de padrão genético, distribuição regional, aparência individual das lesões, achados ultra-estruturais e imunohistoquímicos. De prevalência relativamente comum, exceto a forma juncional progressiva, inicialmente descrita por Gedde-Dahl' , com apenas 18 casos na literatura.
O paciente pode apresentar bolhas nas superfícies externas e internas do corpo, atrofia da pele no dorso das mãos, com perda parcial de impressões digitais e hiperqueratose nas mãos e nos pés. A criança evolui com aparecimento de vários ciclos de formação de bolhas, geralmente com infecção secundária ${ }^{3}$ (Fig. 1-5).

Apesar das manifestações clínicas sugerirem o diagnóstico, ele só é confirmado pelo estudo histopatológico, onde o plano de clivagem indica o subtipo envolvido. A microscopia de luz revela bolhas dermoepidérmicas com conteúdo seroso ou hemorrágico, vasodilatação, infiltrado inflamatório e perda das papilas dérmicas. A microscopia eletrônica de transmissão mostra, na epidermólise bolhosa juncional, ruptura na região de lâmina lúcida da membrana basal e, na forma distrófica, ruptura na lâmina densa. Os hemidesmossomos podem estar em número diminuído ou ausentes ${ }^{1,10}$.

\footnotetext{
* Doutorandos do Programa de Pós-Graduação em Ortodontia - Universidade Federal do Rio de Janeiro/RJ.

** Médica Pediatra. Hospital São Luís. São Paulo - SP.

*** Professora Doutora do Departamento de Ortodontia da Universidade Federal do Rio de Janeiro/RJ.
} 
É necessário que estas crianças sejam acompanhadas por uma equipe multidisciplinar, conduzindo seu desenvolvimento, apesar das dificuldades impostas pela doença. Com este objetivo, serão abordadas as principais características da doença, enfatizando o envolvimento da cavidade bucal e a necessidade de acompanhamento, para diminuir as interferências do desenvolvimento normal da oclusão.

\section{HEREDITARIEDADE}

Em famílias que possuem integrantes próximos ou distantes acometidos por epidermólise bolhosa, o aconselhamento genético é muito importante. Em especial, quando houver o desejo de gravidez, deixando claro o risco de se ter uma criança com a doença.

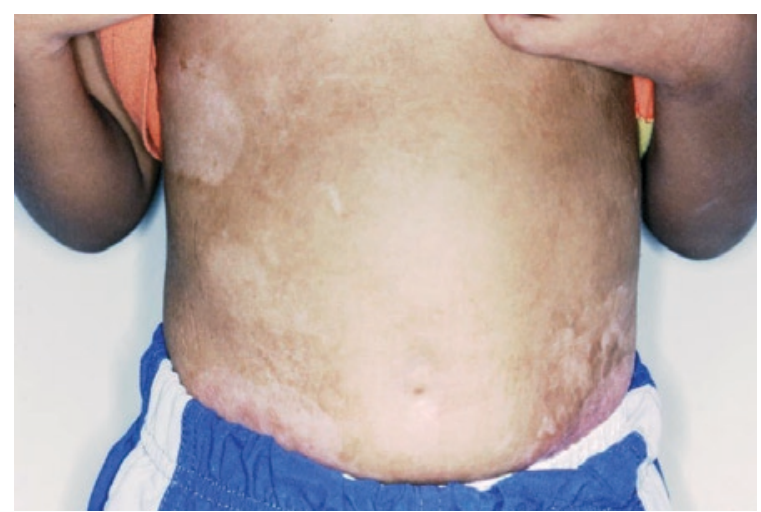

FIGURA 2 - Tecido cicatricial na superfície corporal após ocorrência de boIhas recorrentes.

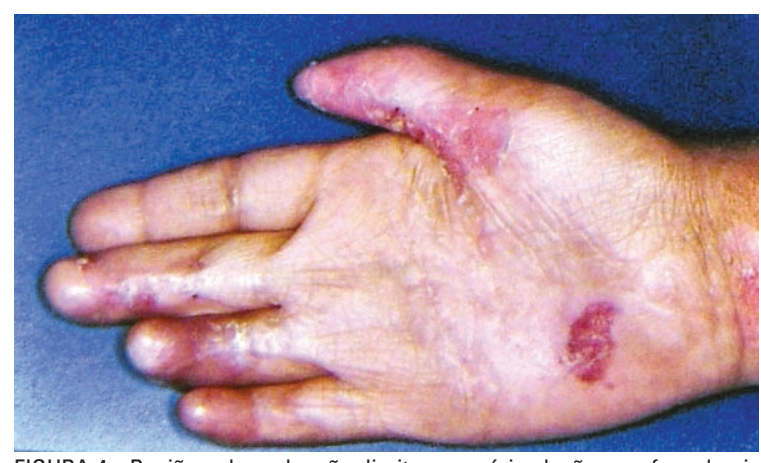

FIGURA 4 - Região palmar da mão direita com várias lesões em fase de cicatrização. Notar extensa fibrose dos tecidos epiteliais, comprometendo 0 desenvolvimento normal dos tecidos ósseo e muscular.
Em geral, formas leves de epidermólise bolhosa (Epidermólise bolhosa simples) são passadas de uma geração para a seguinte, ou seja, de forma autossômica dominante. Nesse caso, o risco de se
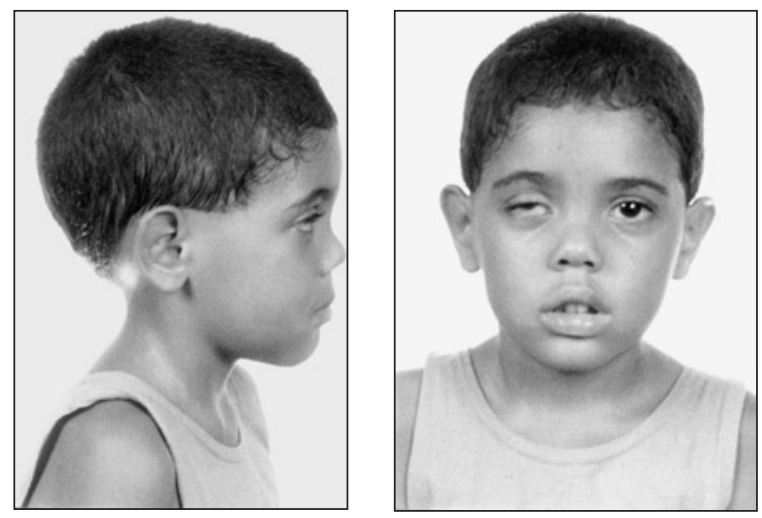

FIGURA 1 - Vista lateral direita e frontal de um paciente com epidermólise bolhosa.

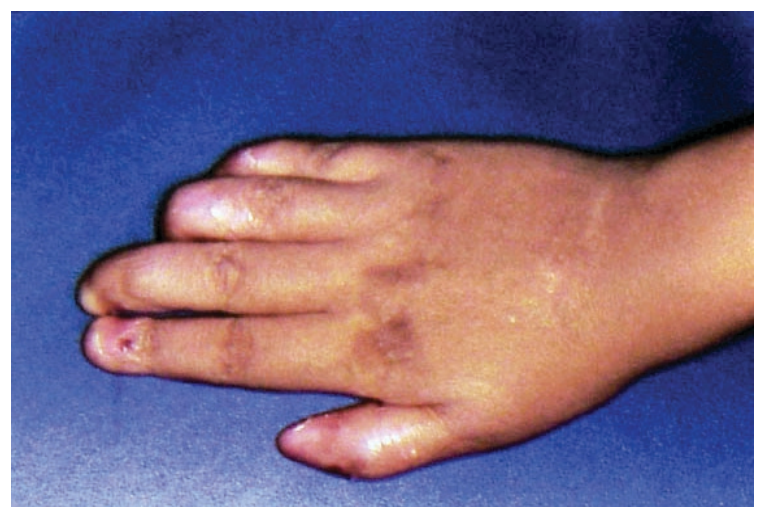

FIGURA 3 - Região dorsal da mão direita, ausência de unhas, desenvolvimento alterado por resistência elástica do epitélio.

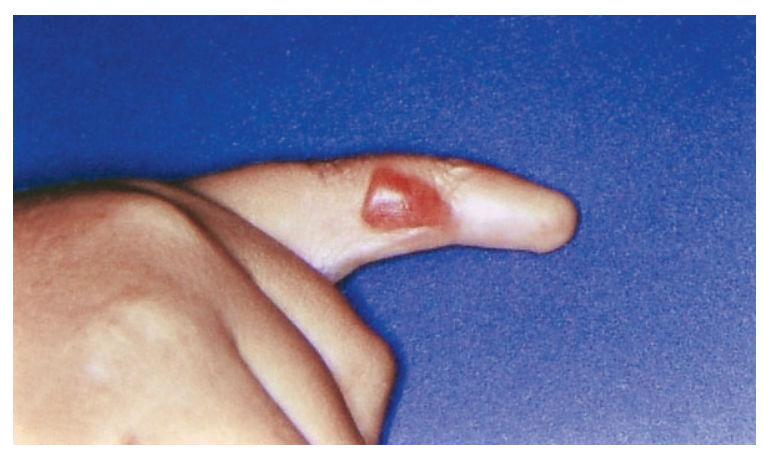

FIGURA 5 - Bolha subepidérmica com conteúdo hemorrágico na região lateral do dedo indicador da mão direita. Notar ausência de unha e presença de tecido cicatricial na extremidade distal. 
ter uma criança acometida, para casais nos quais um dos parceiros é acometido, é de 50\% para cada gravidez. Formas graves de epidermólise bolhosa (Epidermólise bolhosa juncional, anteriormente conhecida como letal) são transmitidas por meio de herança autossômica recessiva. Isso significa que ambos os pais trazem o gene para a doença, embora eles próprios não sejam acometidos. Nesse caso, o risco de se conceber uma criança acometida é de $25 \%$, em cada gestação. Já a forma distrófica tem padrão variável ${ }^{6}$.

\section{ACHADOS CLÍNICOS}

A formação de bolhas existe, geralmente, já ao nascimento ou estas se formam após alguns dias. As manifestações clínicas que iniciam logo após o parto podem, a princípio, ser tratadas como sífilis, sendo diferenciadas, tardiamente, após o período de cicatrização. Em algumas formas de epidermólise bolhosa simples, a doença se apresenta somente na adolescência. $\mathrm{Na}$ idade adulta oferece, freqüentemente, menos problemas, embora a tendência para formação de bolhas permaneça por toda a vida. Nos estágios iniciais, pode-se confundir a doença com infecção da pele e somente quando a formação de bolhas é detida, após demorada cura e formação de cicatrizes, fica claro o diagnóstico. As infecções se apresentam como grande problema, devido à grande superfície de pele exposta ${ }^{9}$.

Distúrbios nos olhos podem acontecer após fricção, que conduz à formação de bolhas nas pálpebras, escoriações da córnea e inflamação ${ }^{3}$.

Deficiência auditiva (disacusia) na epidermólise bolhosa é rara, mas pode acontecer. Acredita-se que a causa seja a formação de bolhas no ouvido interno ${ }^{7}$.

Aderência dos dedos acontece somente com crianças que sofrem de formas graves da doença. A aderência acontece quando ocorre formação constante de bolhas entre os dedos dos pés e das mãos, podendo causar a perda de várias unhas. Para evitar este problema é preconizada ação rápida, aplicando-se gaze com vaselina entre os dedos, com tala especial. Existe a possibilidade de tratamento cirúr- gico ou transplante de pele, entretanto é desaconselhável em crianças, devido ao crescimento ${ }^{10}$.

Crianças que sofrem de grave epidermólise bolhosa distrófica podem ter atraso no crescimento. Com freqüência, a causa pode ser a pequena ingestão alimentar, devida à dificuldade de deglutição, ou anemia. Alguns casos podem ser causados pelo uso de esteróides. A puberdade pode acontecer tardiamente. Apesar disso, muitas crianças podem ter um desenvolvimento normal. A epidermólise bolhosa não está relacionada com a redução da capacidade intelectual ou deficiência mental ${ }^{4}$.

\section{MANIFESTAÇÕES BUCAIS}

$\mathrm{Na}$ cavidade bucal podem ocorrer lesões bolhosas polimórficas nas regiões jugal e vestibular, além de vermelhidão nos lábios. Nas formas juncional e distrófica há suscetibilidade de injúrias, como erosões e eventual desnudamento da língua, além de anquiloglossia e microstomia ${ }^{1,7}$.

A presença de lesões na cavidade bucal e farin-
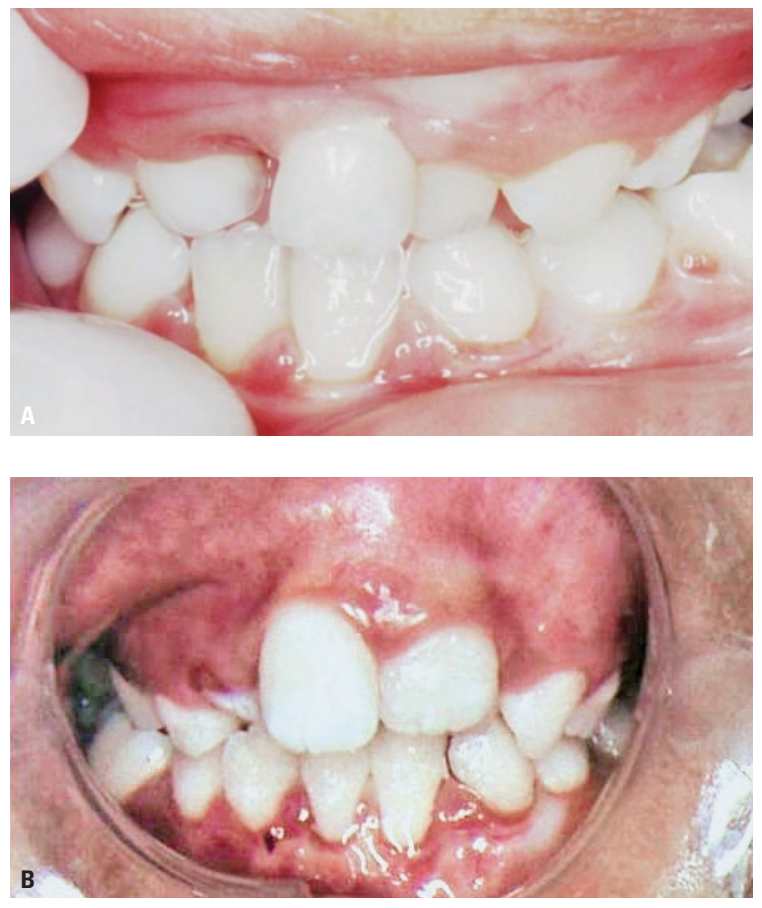

FIGURA 6 - Alteração do desenvolvimento normal da oclusão. A) Lesão ulcerada na papila gengival entre os elementos 74 e 75. B) Mesmo paciente em fase de dentadura mista, aspecto alterado do tecido gengival devido à dificuldade de higienização. 
ge leva à disfonia (dificuldade de fonação), redução da amplitude de abertura de boca, deglutição atípica, desequilíbrio da musculatura e alterações no desenvolvimento dos maxilares ${ }^{9}$.

O acometimento do esmalte dentário, por más formações, é característica da forma juncional. A higienização com uso de escovas dentais pode provocar formação de bolhas e inflamação das gengivas, aumentando o risco à doença cárie (Fig. 6). A extração dentária deve ser evitada nos pacientes com formas graves, pois reabilitações protéticas podem injuriar os tecidos bucais. Da mesma forma, dispositivos ortodônticos favorecem o atrito com tecidos bucais, a formação de bolhas e infecções ${ }^{5,13,16}$.

\section{ALIMENTAÇÃO E DEGLUTIÇÃO}

Especialmente para crianças fortemente ameaçadas por infecções, é muito importante encorajar a amamentação. Com o surgimento de bolhas na cavidade bucal, os movimentos de sucção podem transcorrer lentamente. É desaconselhável a utilização de sonda gástrica em ambiente hospitalar, pois essa pode provocar a formação de bolhas, cicatrizes ou estreitamentos esofágicos ${ }^{5}$.

Enquanto as funções de respiração, sucção e deglutição são inatas e inicialmente controladas de forma reflexa, a mastigação é uma função aprendida e dependente de inúmeros fatores. Esta função só pode ser aprendida a partir do momento em que exista aumento do espaço intrabucal, propiciado pelo crescimento craniofacial, para possibilitar o movimento das estruturas envolvidas. A amamentação é um importante estímulo inicial a este crescimento ${ }^{12}$.

Inicialmente, os movimentos são irregulares e pouco coordenados, como em qualquer outra atividade motora em seu estágio inicial. Conforme a dentadura decídua se completa, os movimentos mandibulares tornam-se mais estáveis. Especialmente durante este período de aprendizagem, a orientação sensorial necessária é fornecida por proprioceptores em diversas estruturas, tais como: terminações nervosas da articulação temporoman- dibular (receptores na ATM), receptores da membrana periodontal, da língua, de toda a mucosa bucal e dos músculos. Nesta fase, é fundamental que a criança tenha a possibilidade de estimulações diferenciadas, tanto pela alimentação com texturas diferentes quanto pelo uso de brinquedos ou objetos que leva à boca. O ato mastigatório é, portanto, uma atividade neuromuscular altamente complexa e considerada como fase preparatória da deglutição. Conseqüentemente, é a partir de eficiente processo mastigatório que a deglutição tem condições de ser efetuada de maneira adequada e sem pressões compensatórias $2,14,15$.

No paciente com epidermólise bolhosa ocorre modificação anatomofisiológica, aumentando as possibilidades de adaptações e de perturbações em todo o sistema, devido ao surgimento de bolhas no aparelho digestivo. Existe grande dificuldade de deglutição, porque esta torna-se dolorosa e pode durar muito tempo. Comumente, bolhas obstruem parcialmente a laringe e o esôfago, podendo levar ao sufocamento e dor. O esôfago pode se curar espontaneamente, permanecendo estreito e com cicatrizes que dificultam o trânsito normal do alimento, ou ser tratado cirurgicamente para solução dos problemas ${ }^{9}$.

A boa alimentação é relevante, pois há perda constante de secreção através da pele, que leva à perda de proteínas e sangue. Pode ser necessário oferecer suplemento protéico e vitamínico. Devese procurar aconselhamento com nutricionistas e pediatras, para monitorar o ganho de peso e o crescimento $^{4,9}$.

A variedade, quantidade e consistência dos alimentos devem ser aumentadas, à medida que a criança cresce e oferece aceitação, favorecendo a fisiologia do aparelho estomatognático, o adequado crescimento craniofacial e o desenvolvimento correto das dentaduras ${ }^{2}$.

\section{TRATAMENTO}

O tratamento deve ser multidisciplinar, porém não existe nenhum tipo específico de terapêutica. 
O uso de Citocinas e Fatores de Necrose Tumoral (Tumor Necrotic Factor) é sugerido no tratamento de redução das bolhas epiteliais e mucosas. Devese evitar traumas cutâneo-mucosos durante atividades de lazer, tomando cuidados com banhos, uso de roupas e calçados com acessórios, entre outros. Infecções secundárias devem ser tratadas, pelo pediatra ou dermatologista, com antimicrobianos tópicos e/ou sistêmicos e dieta rica em proteínas, ferro e zinco. Otorrinolaringologistas devem estar familiarizados com esta patologia, no diagnóstico diferencial de lesões bolhosas das vias aéreas superiores e, também, no diagnóstico das disacusias (dificuldade auditiva). A fisioterapia é muito importante para o reforço das articulações, principalmente quando estas áreas apresentam cicatrizes. $\mathrm{Na}$ articulação temporomandibular, os benefícios dos exercícios fisioterápicos permitem reforço da musculatura, movimentos mandibulares corretos e desenvolvimento equilibrado ${ }^{7}$.

O acompanhamento odontológico é imperativo. A análise funcional deve ser realizada diagnosticando padrões de deglutição, fonação e ausência de hábitos deletérios. Hipotonia muscular e respiração bucal são achados freqüentes nestes pacientes. $\mathrm{O}$ plano de tratamento deve prever cuidados para preservar a integridade do esmalte dentário, por meio de medidas preventivas e controles odontopediátricos periódicos. A criança deve, tão cedo quanto possível, ser habituada a uma rigorosa higiene bucal, que pode ser realizada com uso de cotonete, gaze ou escova dental macia. Creme dental pode ser utilizado a partir do $3^{\circ}$ ano de vida, lembrando que este não deve produzir ardor nas lesões da mucosa, assim como as soluções antimicrobianas utilizadas para reduzir o risco de infecções. A terapia com flúor é imprescindível ${ }^{6,11}$.

A programação de consultas de controle deve respeitar manifestações clínicas e limitações impostas pela doença, entretanto supervisões semestrais do desenvolvimento da oclusão podem diminuir o risco de alterações na dentadura permanente, bem como permitem ao clínico oportunidades de ob- servação da saúde geral do paciente ${ }^{17}$.

Considerações especiais devem ocorrer durante a condução do tratamento ortodôntico interceptativo, como cuidados durante os exames clínicos, devido à limitação de abertura bucal, e lubrificação da mucosa antes de moldagens para confecção de modelos. Caso haja indicação, o planejamento deve sugerir aparelhos que não causem injúrias à mucosa e recomendações durante os períodos de uso, evitando a ocorrência de bolhas e infecções secundárias.

\section{RECOMENDAÇÕES}

As vacinas são extremamente importantes para as crianças com epidermólise bolhosa, devido ao rompimento da barreira epitelial e mucosa de defesa. Vacina contra catapora deve ser ministrada.

O uso de chupetas é contra indicado, por agredir a mucosa bucal e promover maior desequilíbrio neuromuscular. Exercícios fisioterápicos que estimulem a motricidade oral são indicados para o desenvolvimento das bases ósseas e posicionamento dos dentes em harmonia.

Para a prevenção de infecções das vias aéreas, exercícios fisioterápicos especiais para o aparelho respiratório são preconizados.

Tanto quanto possível, as crianças com epidermólise bolhosa devem freqüentar a escola normalmente. Sua inteligência não é afetada pela doença. Professores e colegas devem ser informados que a epidermólise bolhosa não é contagiosa.

A interação com demais especialidades facilita o planejamento de terapias, divide responsabilidades e enriquece o atendimento. 


\title{
Multidisciplinary management of the orthodontic patient with epidermolysis bullosa
}

\begin{abstract}
The term epidermolysis bullosa describes a varied group of hereditary, chronic, non-inflammatory diseases of the skin and mucosa, which are characterized by remarkable skin fragility and reduced resistance after moderate trauma. This disease is classified as simple, junctional or dystrophic form, with at least 23 subtypes, and is a rare disease found in all populations and racial groups, equally affecting males and females. Aim: the authors reports systemic and oral problems associated with epidermolysis bullosa, guiding the multidisciplinary clinical attitudes when the patient needs orthodontic treatment.
\end{abstract}

Key words: Epidermolysis bullosa. Orthodontics. Multidisciplinary management.

\section{REFERÊNCIAS}

1. BIRCHER, A. J.; LANG-MURITANO, M.; PFALTZ, M.; BRUCKNER-TUDERMAN, L. Epidermolysis Bullosa Juncionallis Progressiva in three siblings. Br. J. Dermatol., London, v. 128, no. 4, p. 429-435, Apr. 1993.

2. DE BENEDITTIS, M.; PETRUZZI, M.; FAVIA, G.; SERPICO, R. Oro-dental manifestations in Hallopeau-Siemens-type recessive dystrophic epidermolysis bullosa. Clin. Exp. Dermatol., Oxford, v. 29, no. 2, p. 128-132, Mar. 2004.

3. EADY, R. A. J. The Classification of Epidermolysis Bullosa. In: PRIESTLEY, G. C.; TIDMAN, M. J.; WEISS, J. B., EADY, R. A. J. EB: a comprehensive review of classification, management and laboratory studies. Growthome: Berkshire, 1990. p. 1-9.

4. FEURLE, G. E.; WEISDAUER, H.; BAULDAUF, G.; SCHULTEBRANCS, T.; ANTON-LAMPRECHT, I. Management of esophageal stenosis in recessive dystrophic epidermolysis bullosa. Gastroenterology, New York, v. 87, no. 6, p. 1376-1380, Dec. 1984.

5. FINE, J. D.; EADY, R. A.; BAUER, E. A.; BRIGGAMAN, R. A.; BRUCKNER-TUDERMAN, L.; CHRISTIANO, A.; HEAGERTY, A.; HINTNER, H.; JONKMAN, M. F.; McGRATH, J.; McGUIRE, J.; MOSHELL, A.; SHIMIZU, H.; TADINI, G.; UITTO, J. Revised classification system for inherited epidermolysis bullosa: report of the Second International Consensus Meeting on diagnosis and classification of epidermolysis bullosa. J. Am. Acad. Dermatol., St. Louis, v. 42, no. 6, p. 1051-1066, June 2000.

6. FONSECA, J.; OBADIA, L. Epidermólise bolhosa: recentes avanços. An. Bras. Dermatol., Rio de Janeiro, v. 65, p. 171 174, 1990.

7. GEDDE-DAHL JR., T. Phenotype-genotype correlations in epidermolysis bullosa. Birth Defects Orig. Artic. Ser., New York, v. 7, no. 8, p. 107-117, June 1971.

8. GEDDE-DAHL JR., T. Epidermolysis Bullosa. In: EMERY, A. E. H.; RIMSON, D. L. Principles and practices of medical Genetics. New York: Churchill Livingstone, 1983. v. 1, p. 672-687.

9. GRYBOSKI, J. D.; TOULOUKIAN, R.; CAMPANELLA, R. A. Gastrointestinal manifestations of epidermolysis Bullosa in children. Arch. Dermatol., Chicago, v. 124, no. 5, p. 746-752, May 1988.

10. HABER, R. M.; HANNA, W. Epidermolysis Bullosa progressiva. J. Am. Acad Dermatol, St. Louis, v. 16, no. 1, p. 195-200, Jan. 1987. Pt. 2.
11. KOSTARA, A.; ROBERTS, G. J.; GELBIER, M. Dental maturity in children with dystrophic epidermolysis bullosa. Pediatr. Dent., Chicago, v. 22, no. 5, p. 385-388, Sept./Oct. 2000.

12. MOYERS, R. E.; CARLSON, D. S. Maturação da neuromusculatura orofacial. In: ENLOW, D. H. Crescimento facial. 3. ed. São Paulo: Artes Médicas, 1993. p. 260-271.

13. NOWARK, A. J. Oropharyngeal lesions and their management in epidermolysis bullosa. Arch. Dermatolol., Chicago, v. 124, no. 5, p. 742-745, May 1988.

14. OYEN, O. J. A função mastigatória e o crescimento e desenvolvimento facial. In: ENLOW, D. H. Crescimento facial. 3. ed. São Paulo: Artes Médicas, 1993. p. 272-290.

15. PROFFIT, W. R. Ortodontia contemporânea. 2. ed. Rio de Janeiro: Guanabara Koogan, 1995. p. 195-197.

16. WRIGHT, J. T.; FINE, J. D.; JOHNSON, L. B. Oral soft tissues in hereditary epidermolysis bullosa. Oral Surg. Oral Med. Oral Pathol., St Louis, v. 71, no. 4, p. 440-446, Apr. 1991.

17. WRIGHT, J. T. Comprehensive dental care and general anaesthetic management of hereditary epidermolysis bullosa: a review of fourteen cases. Oral Surg. Oral Med. Oral Pathol., St. Louis, v. 70, no. 5, p. 573-578, Nov. 1990.

Endereço para correspondência

Patrícia Valéria Milanezi Alves

Rua Tonelero, n. 191, apto 806 - Copacabana

CEP: 22.030-000 - Rio de Janeiro / RJ

E-mail: patricia.alves@ortodontia.ufrj.br 